\title{
Cryo Utilities Room Cooling System
}

\author{
G. S. Ball
}

\author{
$01 / 26 / 89$
}

D-Zero Engineering Note \#3740.510.EN-203

Approved:

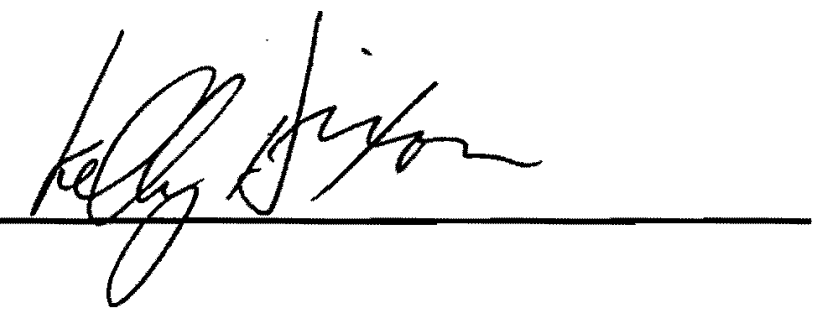


Many of the mechanical equipment failures at the Laboratory are due to the loss of cooling water. In order to insure the proper operating temperatures and to increase the reliability of the mechanical equipment in the D0 Cryo Utilities Room it is necessary to provide an independent liquid cooling system. To this end, an enclosed glycol/water cooling system which transfers heat from two vane-type vacuum pumps and an air compressor to the outside air has been installed in the Cryo Utilities Room.

\section{System Selection}

From the appended list it can be seen that only the Thermal Precision PFC-121-D and Ingersoll-Rand WAC 16 deserve closer investigation based on price. The disadvantages of the WAC 16 are that: it runs a little warmer, it requires more valving to properly install a backup pump, inlet and outlet piping are not included, and temperature and pressure indicators are not included. Its only advantage is that it is $\$ 818$ cheaper than the PFC-121-D. The advantages of the PFC-121-D are that: it has automatic pump switching during shutdown, it has a temperature regulator on one fan control, it has a switch which indicates proper operation, has a sight glass on the expansion tank, and comes with an ASME approved expansion tank and relief valve. For these reasons the Thermal Precision PFC-121-D was chosen.

\section{System Details}

In the past, we have always found the pond water to be muddy and to sometimes contain rocks of greater than $1 / 2$ inch diameter. Thus a system completely dependent on the pond water from the accelerator was deemed unacceptable. A closed system was selected based on its ability to greatly improve reliability, while remaining economical. It is charged with a 50/50 glycol/water mixture capable of withstanding outside temperatures down to $-33^{\circ} \mathrm{F}$. The fluid will be circulated by a totally enclosed air cooled Thermal Precision PFC-121-D pump. The system will be on emergency power and an automatically controlled backup pump, identical to the primary, is available should the main purnp fail. The fan unit is used as a primary cooler and the trim cooler cools the fluid further on extremely hot days. The trim cooler has also been sized to cool the system in the event of a total shutdown provided that the pond water supply has adequate pressure. Due to a broken filter, we found it necessary to install a strainer in the pond water supply line. The expansion tank separates air bubbles, ensures a net positive suction head, protects against surges and over pressurization of the system, and allows 


\section{Status}

All piping has been installed, flushed, charged with the glycolwater mix, and hydrostatically tested to $55 \mathrm{psi}$. The condition of all pumps and flow conditions will be recorded at the PLC. It has been decided not to include the regulator valve in the pond water return line. This valve was designated by the manufacturer to reduce the amount of water flowing through the trim cooler. This is not necessary in our application. There is some concern that the cooling fluid may cool the mechanical equipment too much when they are not operating or during very cold days. This issue will be addressed and the conclusion appended to this engineering note. 


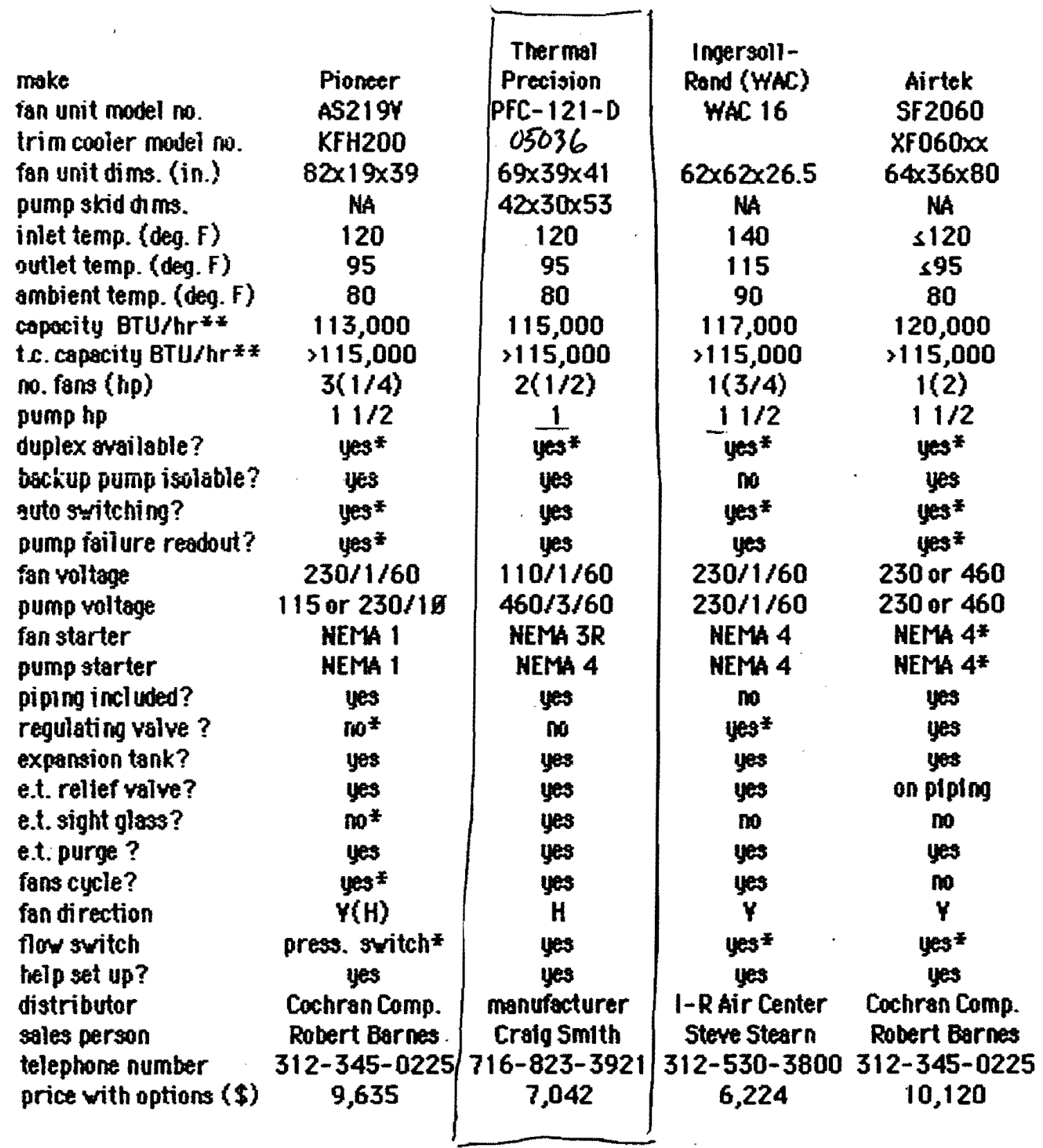

* indicates an optional item

** using a $40 \%$ gl ycal mix 



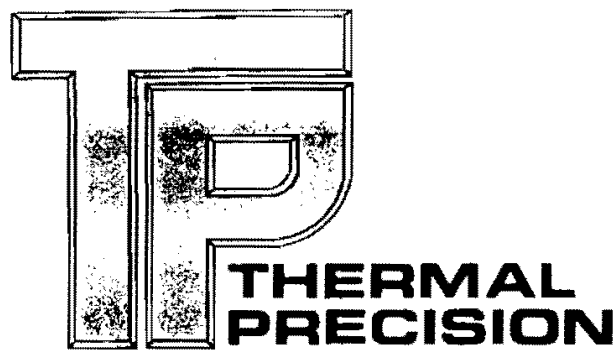

\section{DRY FLUID COOLER}

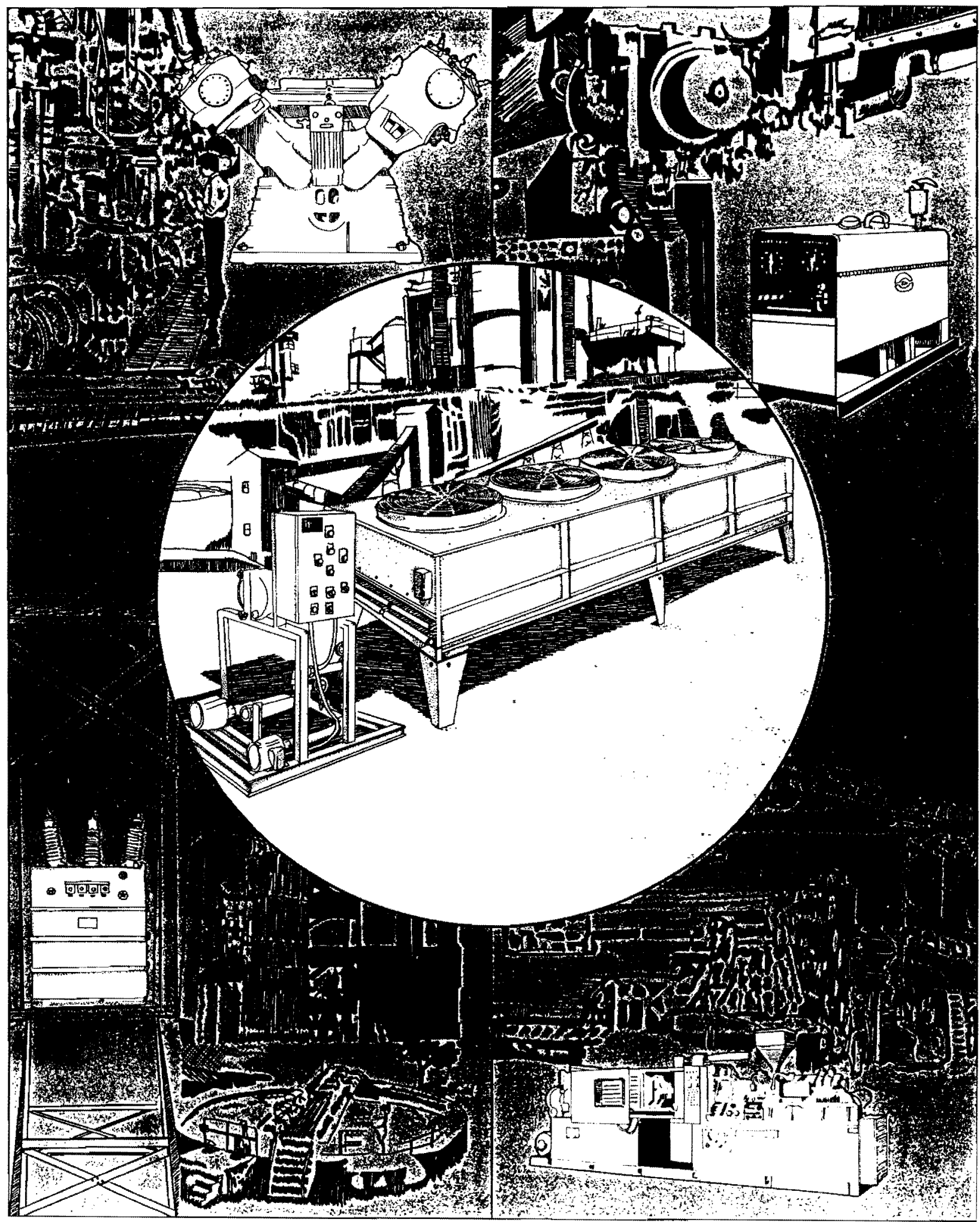



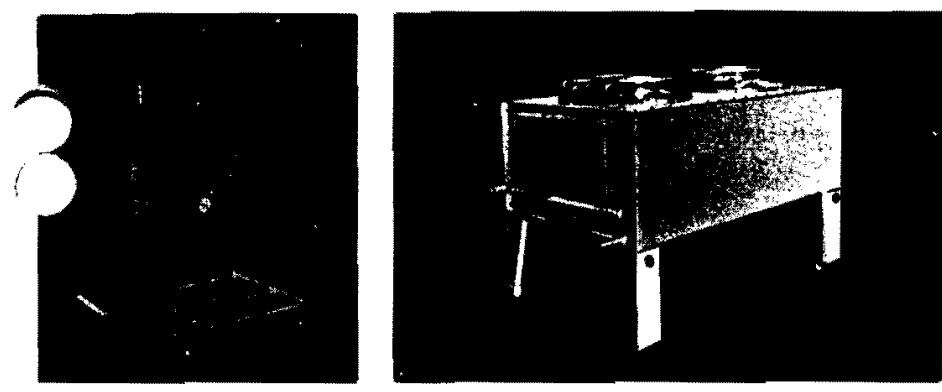

\section{Water - An Endangered National Resource}

Droughts in the Midwest. Bans on lawn sprinkling in the East. Lowering water tables nation-wide. These are all symptoms of our dwindling natural water resources. The end result? An ever-rising price to use and dispose of water.

If you are operating water-cooled equipment in your plant, you are faced with:
- increasing water costs
- sewage discharge taxes
- tightening government regulations
- discharge regulation compliance costs
- scaling and corrosion problems

\section{The Alternative? A THERMAL PRECISION Packaged Fluid Cooler.}

A sealed, pressurized recirculating cooling system consisting of an air-cooled fan-coil unit coupled to a pump station/control module, the Thermal Precision Fluid Cooler is THE answer to your water usage dilemma.

Engineered to match your heat load, the Thermal Precision Fluid Cooler utilizes a continuously circulating water/glycol solution to cool your equipment. This mixture in turn is cooled by ambient air passing over the fan coil unit.

The completely sealed system eliminates scale and corrosion build-up in piping and equipment by eliminating entrained oxygen in the system. The glycol mixture contains corrosion inhibitors for further protection.

As a complete package, the Thermal Precision Fluid Cooler is easily installed at your site. We offer either one complete module for outdoor mountings, or as two separate modules, enabling you to install the pump/control module inside and remote mount the air-cooled heat exchanger outdoors.

Compared to water and sewage expenses, the costs to operate the Thermal Precision Fluid Cooler is miniscule. And through the use of our standard fan cycling sequence control, we make it even more efficient.

Got a critical application which cannot afford to shut down due to lack of cooling water flow? Specify the Thermal Precision Fluid Cooler with optional dual pump arrangement. With this option we provide a manual lead pump selector switch, a pump failure alarm light with remote alarm contacts, automatic switch over tolag pump and warning light advising that a switch-over has occurred.

For difficult temperature situations, or under high ambient conditions, we offer optional trim cooler packages, complete with thermostatic mixing valves. Or, if you prefer, we can provide the Thermal Precision system with evaporative coolers rather than dry fan-coil units.

\section{Feature}

1.) Closed air cooled system

2.) Glycol system

3.) Complete package

4.) Fan cycling
Benefit

No water costs

No sewage cost

No chemical treatment

No scale or corrosion

No freeze up

Low installation cost

Low operating cost

\section{Payback - The Bottom Line}
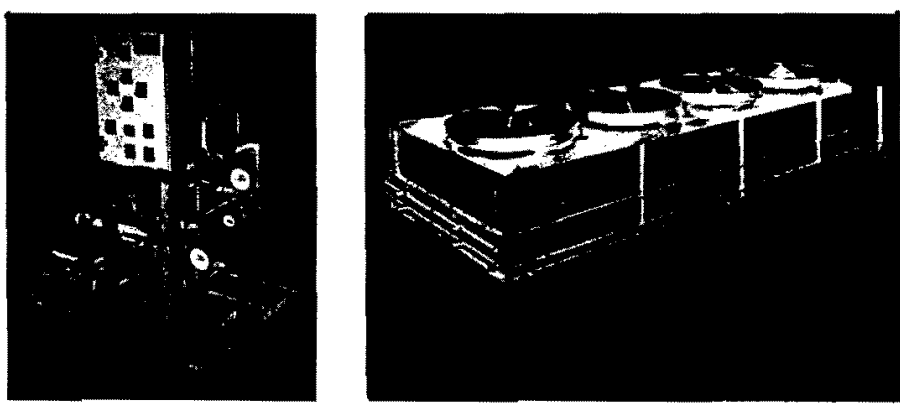

Economic payback on your investment in Thermal Precision equipment is rapid. Directly related to your water and sewer costs, it is easily calculated.

For Example, A heat source using $30 \mathrm{gpm}$ of water, 24 hours per day, 7 days per week with water and sewage costs of $\$ 2.00$ per 1000 gallons = 365 days $\times 24 \mathrm{hrs} \times 60$ minutes $\times 30 \mathrm{gpm} \times \$ 0.002$ per gallon $=\$ 31,536.00$ per year of water \& sewage charges. When compared to the price of a Thermal Precision Fluid Cooler including installation and annual operating cost; you will find payback will occur within as little as 6 months.

Our representative will be pleased to prepare a payback analysis for your specific applications or:

\section{Calculate Your Own Payback}

\begin{tabular}{|c|c|c|c|c|c|c|}
\hline & GPM & $X_{\text {Per Year }}^{\text {Days Work }}$ & $x_{\text {Per Day }}^{\text {Hours Worked }}$ & $X 60$ (Minutes) & $\times \begin{array}{l}\text { Water \& Sewage } \\
\text { Cost Per Gallon }\end{array}$ & $\begin{array}{l}\text { Annual Water } \\
\& \text { Sewage Cost }\end{array}$ \\
\hline Your & & & & 60 & & \\
\hline Application & & & & 60 & & \\
\hline
\end{tabular}


Dry Fluid Cooler Selection

\begin{tabular}{|c|c|c|c|c|c|c|c|c|c|c|c|c|}
\hline \multirow{2}{*}{\multicolumn{4}{|c|}{$\begin{array}{l}\text { Fluid Out - To Heat Load Degrees F } \\
\text { Fluid In - From Heat Load Degrees F }\end{array}$}} & \multicolumn{9}{|c|}{105} \\
\hline & & & & & & & 125 & & & & & \\
\hline \multicolumn{4}{|c|}{$\%$ Glycol } & \multicolumn{3}{|c|}{20} & \multicolumn{3}{|c|}{$30-40$} & \multicolumn{3}{|c|}{50} \\
\hline \multicolumn{2}{|c|}{ Ambient } & \multicolumn{2}{|c|}{ Degrees $F$} & 90 & 95 & 100 & 90 & 95 & 100 & 90 & 95 & 100 \\
\hline HP & MBH & GPM $^{1}$ & PUMP & \multicolumn{9}{|c|}{ PFC MODEL } \\
\hline 20 & 55 & 5 & 1 & 116 & 116 & 121 & 116 & 117 & 117 & 116 & 117 & 123 \\
\hline 25 & 70 & 7 & 1 & 117 & 117 & 123 & 117 & 117 & 121 & 117 & 121 & 125 \\
\hline 30 & 85 & $\overline{8}$ & 1 & 121 & 121 & 123 & 121 & 121 & 123 & 121 & 123 & 125 \\
\hline 40 & 110 & 11 & 1 & 121 & 123 & 134 & 121 & 123 & 134 & 121 & 125 & 134 \\
\hline 50 & 140 & 14 & 1 & 123 & 125 & 137 & 123 & 134 & 137 & 125 & 134 & 137 \\
\hline 60 & 170 & 17 & 2 & 125 & 134 & 225 & 125 & 134 & 225 & 125 & 134 & 225 \\
\hline 75 & 210 & 21 & 2 & 134 & 137 & 227 & 134 & 137 & 227 & 134 & 137 & 227 \\
\hline 100 & 280 & 28 & 2 & 137 & 227 & 231 & 137 & 229 & 234 & 225 & 229 & 237 \\
\hline 125 & 350 & 35 & 2 & 227 & 231 & 237 & 227 & 231 & 326 & 227 & 231 & 326 \\
\hline 150 & 420 & 42 & 3 & 229 & 237 & 326 & 231 & 237 & 328 & 229 & 237 & 328 \\
\hline 200 & 560 & 56 & 3 & 234 & 325 & 331 & 237 & 326 & 333 & 237 & 328 & 333 \\
\hline 250 & 700 & 70 & 3 & 325 & 328 & 336 & 326 & 328 & 336 & 326 & 331 & 339 \\
\hline 300 & 840 & 84 & 5 & 328 & 331 & 339 & 328 & 333 & 342 & 328 & 333 & 342 \\
\hline 350 & 980 & 98 & 5 & 328 & 333 & 342 & 331 & 336 & 342 & 333 & 336 & 342 \\
\hline 400 & 1120 & 112 & 5 & 333 & 336 & 342 & 333 & 339 & 345 & 333 & 339 & 345 \\
\hline 500 & 1400 & 140 & 7.5 & 336 & 342 & 361 & 339 & 342 & 361 & 339 & 342 & 361 \\
\hline 600 & 1680 & 168 & 7.5 & 342 & 342 & 361 & 342 & 345 & 364 & 342 & 361 & 364 \\
\hline
\end{tabular}

1. Fows are basis water and will increase with glycol concentration.

2. Pump selection based on 100 PDF. Refer to Selection Manual for proper sizing.

Flow Schematic

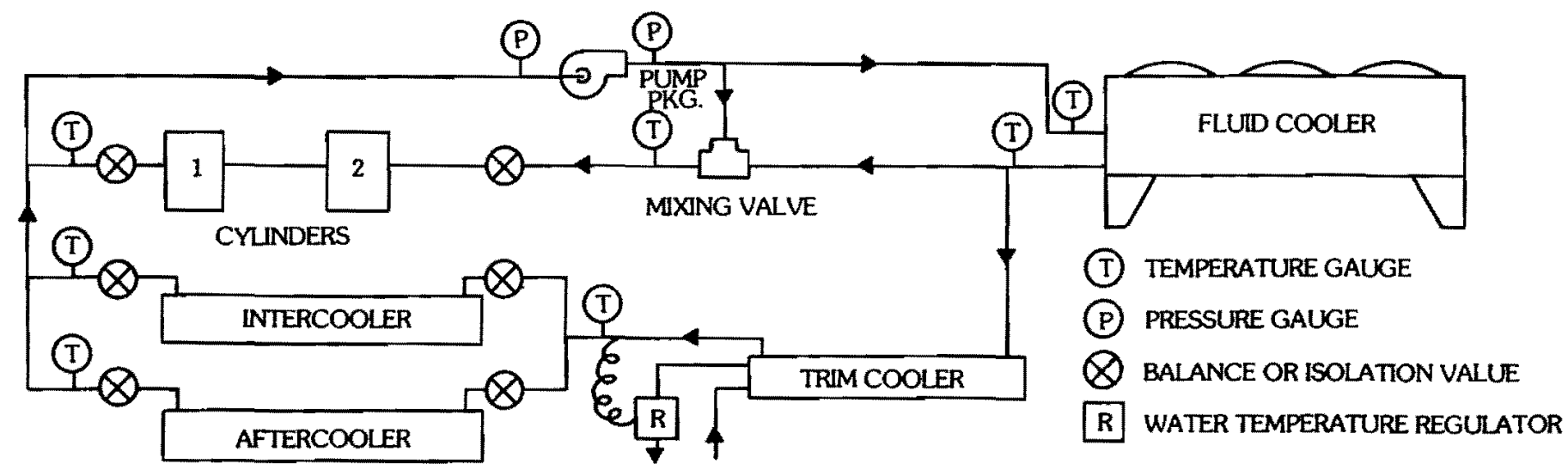

\section{Standard Equipment}

Heat Exchanger Colls - Direct drive fans (Series 100 \& 200)

- Belt drive fans (Series 300)

- Thermal overload protection

- Fan cycling controls (Multi-fan units only)

- NEMA 3R control panels

- Unit cabinets fabricated of either heavy gauge corrosion-resistant aluminum or galvanized steel

- All legs of galvanized steel

- Fans dynamically \& statically

balanced

- Fan guards of heavy gauge close mesh steel wire

- Pre-piped and pre-wired to single point connections
Pump Station (simplex or duplex)

- Close coupled centrtfugal pump(s)

with mechanical seal

- Pump discharge check valve(s)

- Suction and discharge tsolation and

flow control values

- Flow switch

- Pressure and temperature gauges

- Expansion/fill tank with air purge

line, safety valve, isolation value and fluid level sight glass

- Fabricated steel base plate

- Pre-piped and pre-wired to single point connection
Pump Station (con't)

- NEMA 4 control enclosure.

housing:

- Full voltage magnetic motor starters

- 120 volt control transformer (fused)

- Hand-off-auto selector switch

- Power-on light

- Pump run light(s)

- Pump failure lights with contacts for remote alarm

- Terminal strip for connection to heat exchangers coil controls
Optional

- Dual Pump

- Thermostatic Mixing Values

- Trim Coolers

- Water Make Ub System

- Rernote Control Panels

- Nerna, 12, 4x, 7

- TEFC Motors

- Audible Alarm

- Evaporative Coolers

- Heat Recovery Units

- Custom Packaging

Specifications subject to change without notice. 


\section{PFC Cooler}
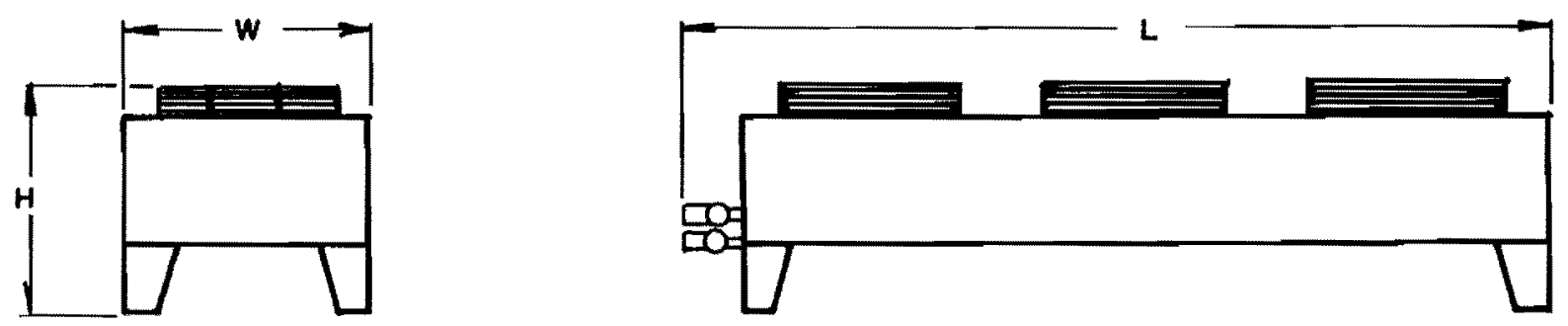

\begin{tabular}{|c|c|c|c|c|c|c|c|c|c||c|c|c|c|c|}
\hline $\begin{array}{c}\text { MODEL } \\
\text { (PFC) }\end{array}$ & L & W & H & $\begin{array}{c}\text { WT. } \\
\text { (LBS.) }\end{array}$ & $\begin{array}{c}\text { MODEL } \\
\text { (PFC) }\end{array}$ & L & W & H & $\begin{array}{c}\text { WT. } \\
\text { (LBS.) }\end{array}$ & $\begin{array}{c}\text { MODEL } \\
\text { (PFC) }\end{array}$ & L & W & H & $\begin{array}{c}\text { WT. } \\
\text { (LBS.) }\end{array}$ \\
\hline 114 & 39.312 & 39.062 & 41.25 & 200 & 227 & 119.75 & 44.562 & 50 & 570 & 328 & 121 & 85.875 & 62.5 & 1610 \\
\hline 116 & 39.312 & 39.062 & 41.25 & 225 & 229 & 119.75 & 44.562 & 50 & 590 & 331 & 176 & 85.875 & 62.5 & 2110 \\
\hline 117 & 39.312 & 39.062 & 41.25 & 245 & 231 & 174.75 & 44.562 & 50 & 840 & 333 & 176 & 85.875 & 62.5 & 2380 \\
\hline 121 & 69.312 & 39.062 & 41.25 & 300 & 234 & 174.75 & 44.562 & 50 & 850 & 336 & 176 & 85.875 & 62.5 & 2460 \\
\hline 123 & 69.312 & 39.062 & 41.25 & 340 & 237 & 174.75 & 44.562 & 50 & 880 & 339 & 176 & 85.875 & 62.5 & 2580 \\
\hline 125 & 69.312 & 39.062 & 41.25 & 380 & 322 & 121 & 85.875 & 62.5 & 1280 & 342 & 231 & 85.875 & 62.5 & 3370 \\
\hline 134 & 99.312 & 39.062 & 41.25 & 500 & 324 & 121 & 85.875 & 62.5 & 1340 & 345 & 231 & 85.875 & 62.5 & 3450 \\
\hline 137 & 99.312 & 39.062 & 41.25 & 550 & 325 & 121 & 85.875 & 62.5 & 1370 & 361 & 341 & 85.875 & 62.5 & 4995 \\
\hline 225 & 119.75 & 44.562 & 50 & 560 & 326 & 121 & 85.875 & 62.5 & 1540 & 364 & 341 & 85.875 & 62.5 & 5115 \\
\hline
\end{tabular}

\section{Simplex Pump Package}
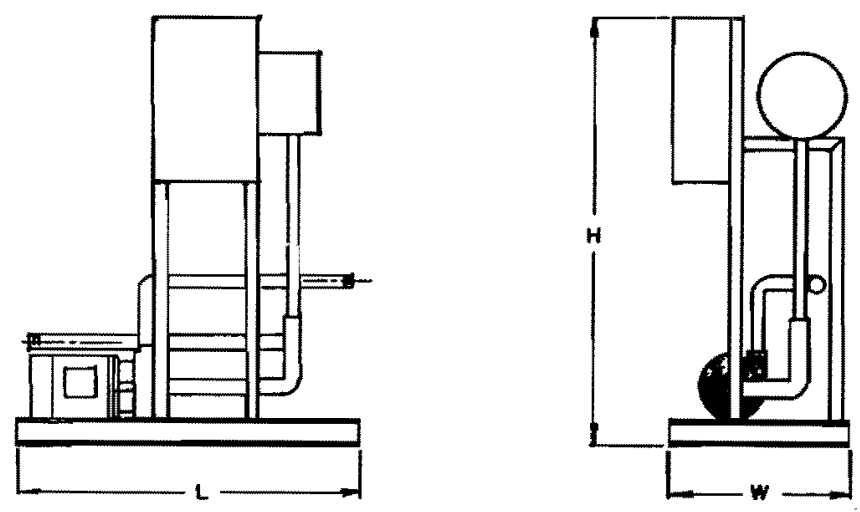

\begin{tabular}{|c|c|c|c|c|}
\hline $\begin{array}{c}\text { MODEL } \\
\text { (HP) }\end{array}$ & $\mathbf{L}$ & $\mathbf{W}$ & $\mathbf{H}$ & $\begin{array}{c}\mathbf{W T} \\
\text { (LBS.) }\end{array}$ \\
\hline 1 & 42 & 24 & 53 & 450 \\
\hline 2 & 42 & 24 & 53 & 465 \\
\hline 3 & 48 & 28 & 53 & 500 \\
\hline 5 & 48 & 28 & 53 & 520 \\
\hline 7.5 & 62 & 30 & 63.5 & 660 \\
\hline 10 & 62 & 30 & 63.5 & 690 \\
\hline 15 & 62 & 30 & 63.5 & 850 \\
\hline
\end{tabular}

\section{Duplex Pump Package}
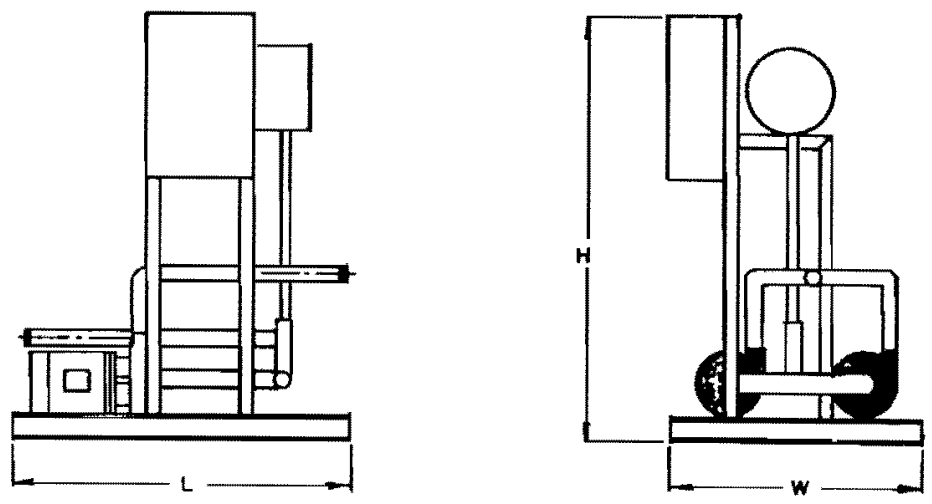

$\rightarrow$\begin{tabular}{|c|c|c|c|c|}
\hline $\begin{array}{c}\text { MODEL } \\
(\mathrm{HP})\end{array}$ & $\mathbf{L}$ & $\mathbf{W}$ & $\mathbf{H}$ & $\begin{array}{c}\text { WT: } \\
(\mathbf{L B S} .)\end{array}$ \\
\hline 1 & 42 & 30 & 53 & 560 \\
\hline 2 & 42 & 30 & 53 & 575 \\
\hline 3 & 48 & 36 & 53 & 730 \\
\hline 5 & 48 & 36 & 53 & 760 \\
\hline 7.5 & 62 & 40 & 63.5 & 1200 \\
\hline 10 & 62 & 40 & 63.5 & 1310 \\
\hline 15 & 62 & 40 & 63.5 & 1500 \\
\hline
\end{tabular}

All Dimensions Are In Inches 


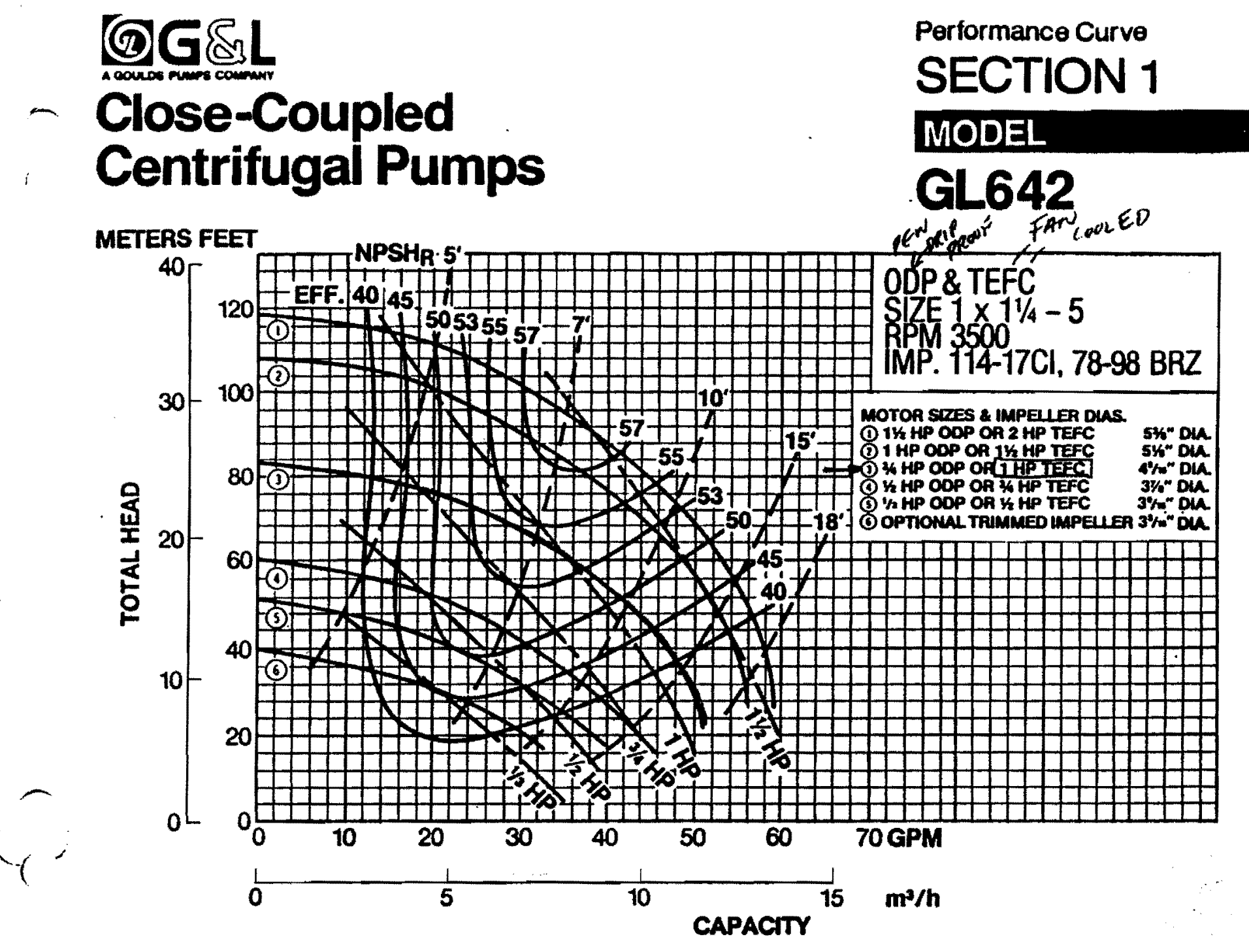




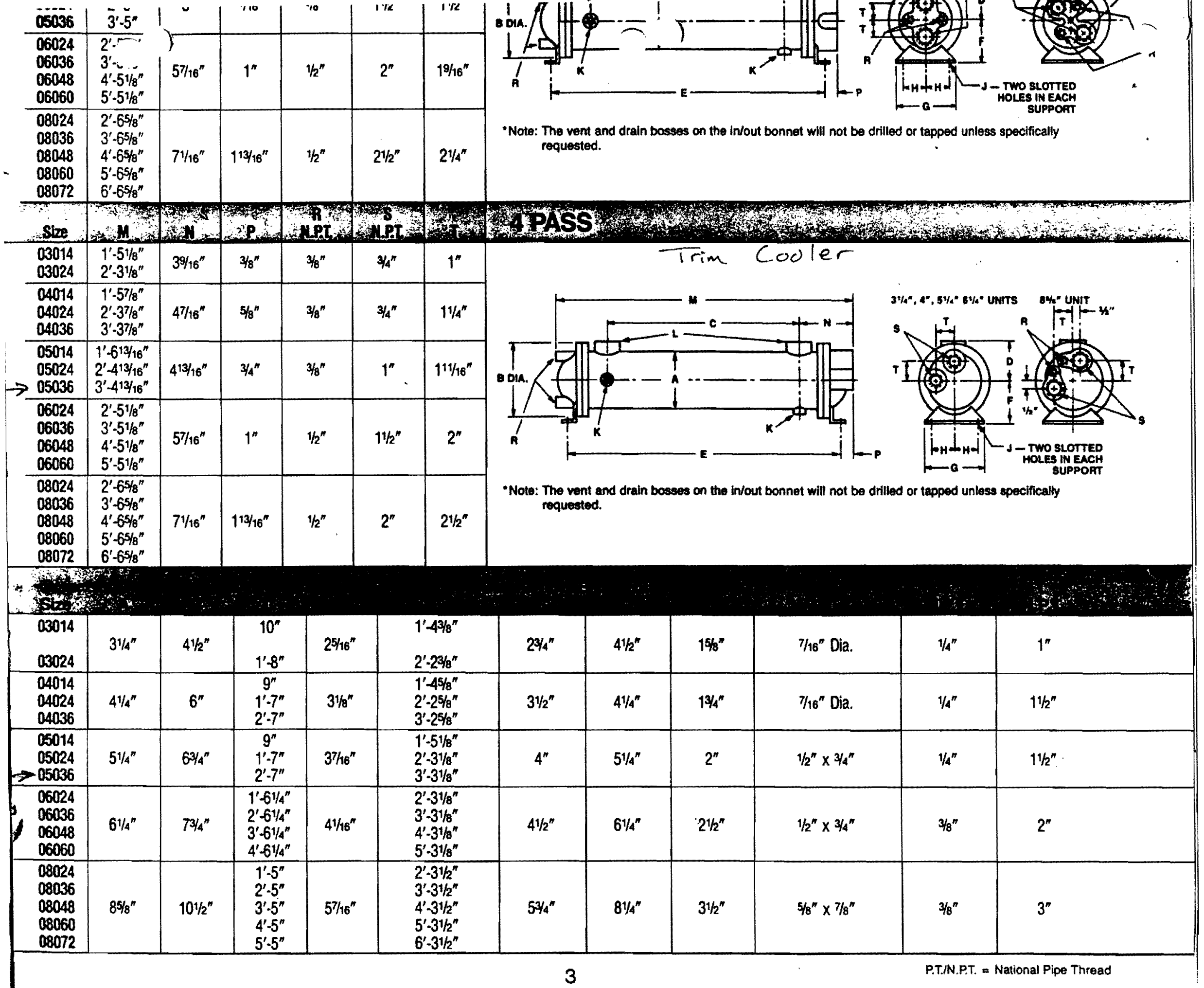

